\title{
Evaluation of the Ordered-Subset Transmission (OSTR) Algorithm for Transmission Imaging on SPECT Systems Using Axially Overlapping Cone-Beams
}

\author{
B. Feng, Member, IEEE, J. A. Fessler, Fellow, IEEE, P. H. Pretorius, Associate Member, IEEE,
} R. D. Beach, Senior Member, IEEE, G. L. Zeng, Senior Member, IEEE, and M. A. King, Senior Member, IEEE

\begin{abstract}
Cone-beam transmission imaging employing medium-energy photons which penetrate parallel-hole collimators can be used to rapidly estimate attenuation maps for use in reconstruction of cardiac SPECT images. Such a transmission imaging geometry offers the advantage of eliminating the need to mechanically move the point-sources during imaging, and enables fast sequential transmission scans. The axial extent over which artifact-free attenuation maps can be reconstructed is limited by the cone-beam geometry and point-source collimation. We investigated irradiation of a single head by multiple point-sources whose asymmetric cone-beam fields overlap in the axial direction as a method of extending the axial coverage of the patient. This study reports on the development and testing of a penalized-likelihood algorithm for transmission reconstruction of overlapping asymmetric cone-beams. We evaluated this algorithm and optimized the reconstruction parameters through MCAT phantom simulations. We then and applied the algorithm to transmission measurements of an anthropomorphic phantom. The experimental work consisted of performing a series of flood and transmission measurements on the anthropomorphic phantom with shifted axial locations of point-sources. We summed the projection data from individual measurements to simulate the projection data for a multiple point-source system. With the proposed penalized-likelihood algorithm, the full axial extent $(20.5 \mathrm{~cm})$ of the anthropomorphic phantom was reconstructed for the overlapping cone-beam geometry with two point-sources per camera head.
\end{abstract}

Index Terms-OSTR, overlapping cone-beams, transmission imaging.

\section{INTRODUCTION}

W E have shown that cone-beam transmission imaging using point-source emitted medium-energy photons that penetrate the parallel-hole collimators on opposed camera-heads can be used to rapidly estimate attenuation maps

Manuscript received October 19, 2004; revised March 22, 2006. This work was supported by Grant HL50349 from the National Heart, Lung, and Blood Institute. The contents are solely the responsibility of the authors and do not necessarily represent the official views of the National Institutes of Health.

B. Feng, P. H. Pretorius, R. D. Beach, and M. A. King are with the Department of Radiology, University of Massachusetts Medical School, Worcester, MA 01655 USA (e-mail: Bing.Feng@umassmed.edu).

J. A. Fessler is with the Department of Electrical Engineering and Computer Science, University of Michigan, Ann Arbor, MI 48109 USA (e-mail: fessler@umich.edu).

G. L. Zeng is with the Department of Radiology, University of Utah, Salt Lake City, UT 84108 USA (e-mail: larry@ucair.med.utah.edu).

Digital Object Identifier 10.1109/TNS.2006.873709

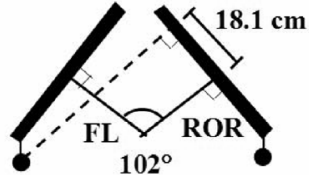

$F L=78.1 \mathrm{~cm}, \mathrm{ROR}=30 \mathrm{~cm}$

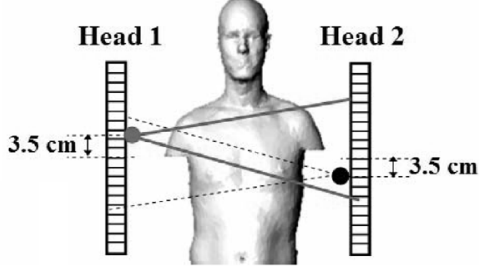

Fig. 1. The transaxial (left) and axial (right) views of the dual-planar conebeam geometry based on the IRIX Beacon transmission system. There is one point-source attached to each head which irradiates the opposed head. The pointsources are displaced axially for wider coverage in the axial direction.

for use in reconstruction of cardiac SPECT images [1]-[4]. Such a transmission imaging geometry offers the advantage of eliminating the need to mechanically move the point-sources during imaging, and enables fast sequential transmission scans. With the help of Philips Medical Systems, we modified the Beacon transmission device [5] on the IRIX SPECT system in our clinic to perform dual-planar [6]-[8] asymmetric cone-beam transmission imaging. This was achieved by positioning the point-source for each of the two heads employed in transmission imaging at a specified axial location before the scan and keeping this location fixed during the scan (Fig. 1). The two Ba-133 point-sources were separated axially to cover a wider axial extent of the object, which resulted in the dual-planar trajectories of the sources in transmission imaging.

Ba-133 is a multiple-energy photon emitter. The energy window employed for transmission imaging was centered at $356 \mathrm{keV}$ with $15 \%$ width. There are two emitted gamma rays within this energy range (356 keV with abundance of $62 \%$, and $384 \mathrm{keV}$ with an abundance of $8.9 \%$ ). These medium-energy photons penetrate the septa of the parallel-hole collimators during transmission imaging. The point-sources are axially collimated because the Beacon system scans the point-sources to cover the full dimension of the camera heads. Based on the flood pattern of a single point-source (Fig. 2), we optimized the axial offset between the two point-sources through experiment measurements of the anthropomorphic phantom and determined that the optimal offset was about $7 \mathrm{~cm}$ [2], which resulted in the artifact-free reconstruction with maximum axial coverage. Using this dual-planar cone-beam geometry, transmission acquisition of a patient can be performed within 40 seconds on the IRIX Beacon system [3]. We determined [3] the 

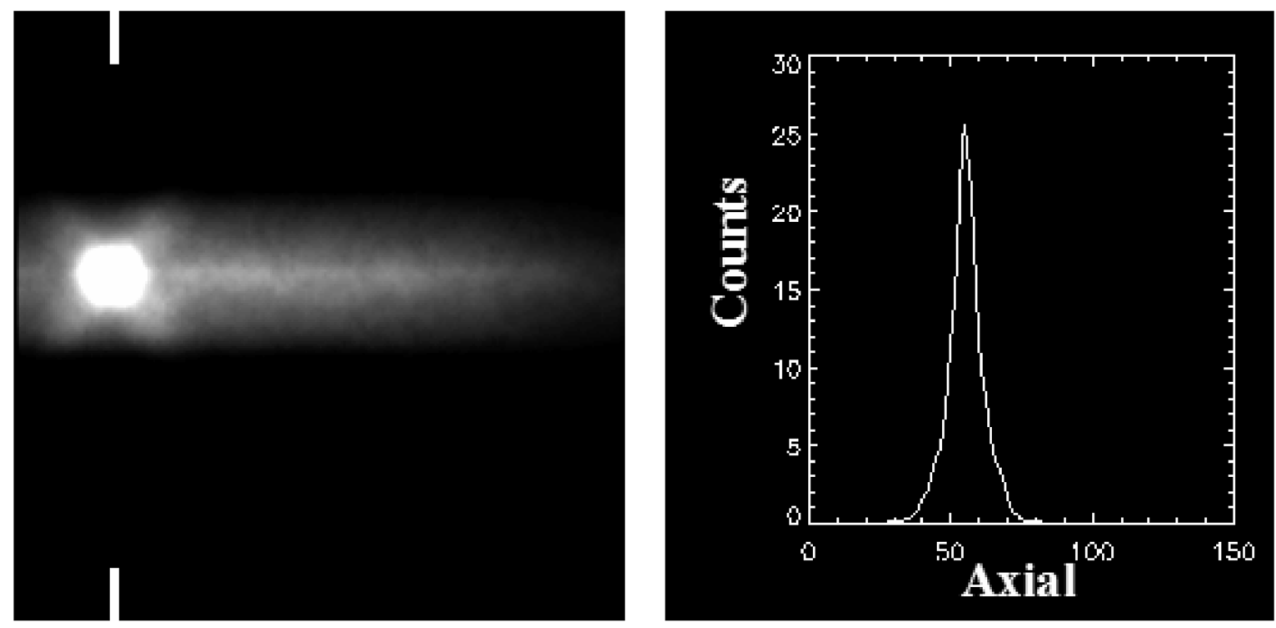

Fig. 2. (Left) Transmission flood from one camera head for the dual-planar cone-beam geometry (the modified Beacon). (Right) The axial profile at the level indicated by the vertical bars on the flood is plotted.



$\mathrm{FL}=78.1 \mathrm{~cm}, \mathrm{ROR}=30 \mathrm{~cm}$

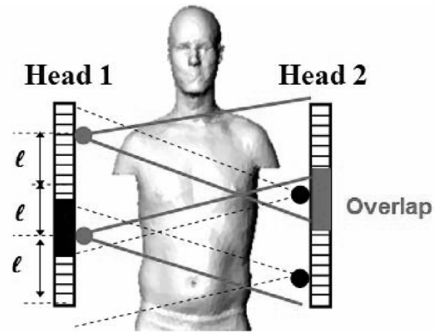

Fig. 3. The transaxial (left) and axial (right) views of the overlapping conebeam geometry investigated. FL-the focal length; ROR - the radius of rotation. $\ell$ is the axial separation between the two neighboring sources that irradiate different detectors. The geometric parameters are adopted from the IRIX Beacon transmission system.

axial extent over which artifact-free attenuation maps can be reconstructed with this dual-planar geometry is limited to $\sim 15$ $\mathrm{cm}$ by the cone-beam geometry and point-source collimation. Though this axial coverage is adequate for cardiac SPECT, it is not adequate for other applications like bone-SPECT.

One way to increase the axial coverage is to open up the source collimation. This is ultimately limited by the incomplete sampling of the cone-beam geometry employed with circular rotation of the sources as one moves farther away from the central plane of the irradiation field, and the reduced ability of the photons to penetrate the opposed collimator as the angle of incidence increases. Herein we therefore investigated irradiation of gamma-camera heads by multiple stationary point-sources where the asymmetric cone-beam fields overlap in the axial direction as a method of extending the axial patient coverage. This geometry is illustrated in Fig. 3.

\section{METHODS}

\section{A. Ordered-Subset Transmission (OSTR) Algorithm Applied to the Case of Overlapping Cone-Beams}

The ordered-subset transmission (OSTR) algorithm was developed by Erdoğan and Fessler [9], and models the Poisson noise in transmission imaging. A penalty term can be easily incorporated into the OSTR algorithm to penalize the roughness of the attenuation map [9]. The OSTR algorithm is the ordered-subset version of the Separable Paraboloidal Surrogates (SPS) Algorithm [10], which is a monotonic algorithm that converges to the global maximum of the penalized likelihood if the penalized likelihood is concave. Otherwise it converges to a local maximum. The OSTR algorithm accelerates the convergence over the SPS algorithm by using subsets.

We have shown that the penalized OSTR algorithm (with an edge-preserving penalty function as described later in equations 1 and 2 below) outperforms nonpenalized algorithms in reconstructing the extremely low-count $(\sim 1.2 \mathrm{M})$ transmission data acquired during a 40-second scan of an anthropomorphic phantom with the cone-beam geometry shown in Fig. 1 [3]. Yu and Fessler [11] adapted the OSTR algorithm to overlapping irradiation beams, and applied it to the multiple-line-source case. As a prerequisite of this algorithm, the flood pattern should be known for each individual transmission source. This algorithm for overlapping beams can be directly applied to the geometry of overlapping cone-beams with only modifications to the projector/backprojector. In the case of overlapping cone-beams, a separate transmission flood is required for each point-source. In the process of reconstruction, the overlapped transmission counts are iteratively sorted for each point-source according to this algorithm which is based on the Poisson statistical model.

To reconstruct smooth attenuation maps we included a roughness penalty

$$
R(\boldsymbol{\mu})=\frac{1}{2} \sum_{j=1}^{N_{p}} \sum_{k=1}^{N_{p}} \omega_{j k} \psi\left(\mu_{j}-\mu_{k}\right)
$$

where $\boldsymbol{\mu}$ is a vector describing the three-dimensional distribution of the attenuation coefficients, $\mu_{j}$ and $\mu_{k}$ are values of the attenuation coefficient at voxels $\mathrm{j}$ and k, respectively, $N_{p}$ is the number of voxels, $\omega_{j k}=\omega_{k j}$ is the weight depending the relative position between voxels $\mathrm{j}$ and $\mathrm{k}$, and $\psi$ is the potential function which gives a cost to $\mu_{j}-\mu_{k}$. We assigned $\omega_{j k}=1$ to the nearest neighboring voxels, $\omega_{j k}=0.5$ to neighboring voxels 
which are separated by a distance of $\sqrt{2}$ pixels, and $\omega_{j k}=0.25$ to neighboring voxels which are separated by a distance of $\sqrt{3}$ pixels, and $\omega_{j k}=0$ otherwise. The potential function adopted here is of the form

$$
\psi(t)=\beta \delta^{2}\left(\sqrt{1+t^{2} / \delta^{2}}-1\right),
$$

where $t=\mu_{j}-\mu_{k}, \beta$ is a scaling factor which will be determined through the MCAT phantom simulations, and $\delta$ is the threshold. In the case that the difference of the attenuation coefficient between the neighboring voxels is larger than the threshold (for $t>\delta$ ), which is likely near the edge of the object, $\psi(t)$ is approximately linear with $t$, otherwise $\psi(t)$ is approximately a quadratic of $t$. Thus this penalty prior is edge-preserving. The threshold $\delta$ was empirically chosen as $0.0025 \mathrm{~cm}^{-1}$, which is about $1 / 20$ of the attenuation coefficient of lungs at $140 \mathrm{keV}$. To generate the separable paraboidal surrogate function [11] of $R(\boldsymbol{\mu})$, we adopted Huber's approach [12] and used the maximum curvature.

\section{B. Simulation of Overlapping Cone-Beam Projections Using MCAT Phantom Simulations}

To increase the axial coverage of the cone-beam geometry, imagine that we couple two identical dual-planar cone-beam systems with the $7 \mathrm{~cm}$ axial offset [2] between sources on opposing heads (i.e., $14 \mathrm{~cm}$ between sources on the same head). This leads to the proposed overlapping cone-beam geometry as shown in Fig. 3 with $\ell=7 \mathrm{~cm}$, where $\ell$ is the axial separation between the two neighboring sources that irradiate different detectors. We chose $7 \mathrm{~cm}$ as the optimum separation between two neighboring sources on the different cameras, as in the case of the dual-planar cone-beam system (Fig. 1) [2]. The resultant overlapping cone-beam geometry consists of a total of $4 \mathrm{Ba}-133$ point-sources (two per head), which are deployed axially at $-10.5,-3.5,3.5,10.5 \mathrm{~cm}$, respectively to the center of the camera filed-of-view (FOV). The gantry angle tested in this study is 102 degrees between two heads hosting the point-sources, which is the same gantry angle used on the IRIX SPECT system with the Beacon transmission device deployed.

To challenge the OSTR algorithm in regards to reconstructing overlapping transmission fields, we also performed simulations of the MCAT phantom with a severely overlapping cone-beam geometry. In this geometry the axial separation between the two neighboring sources that irradiate different detectors was set to $2 \mathrm{~cm}(\ell=2 \mathrm{~cm})$. Thus the separation between two point-sources irradiating the same head was $4 \mathrm{~cm}$.

The measured detected irradiation fields of solely the sources with no objects interposed between the source and camera (transmission floods) for these two overlapping cone-beam geometries (i.e., $\ell=7 \mathrm{~cm}$ and $\ell=2 \mathrm{~cm}$ ) are shown in Fig. 4 . These were measured on our IRIX SPECT system with a 4-minute acquisition which employed a $15 \%$ energy window centered at $356 \mathrm{keV}$. A pixel size of $0.416 \mathrm{~cm}$ was used in acquisition of the floods. To simulate 1-minute 360-degree acquisitions with these two overlapping cone-beam geometries $(\ell=2 \mathrm{~cm}$ and $\ell=7 \mathrm{~cm}$ ), we simulated the transmission projections of the MCAT phantom starting with the measured floods and employing a ray-driven numerical projector. We cast rays through the object and weight them by the appropriate measured transmission flood. Neither scatter in the object nor in the collimator septa was modeled. Septa penetration was included in the simulation only by using the transmission floods measured on the modified Beacon system, which was an approximation that models the attenuation of the primary photon flux by the septa but not scatter. The total flood counts simulated was that of the 4-minute flood acquired on the IRIX which was approximately $3.6 \mathrm{M}$ on each of the two detectors. Both noise-free projections and projections with Poisson noise were generated for the severely overlapping cone-beam geometry $(\ell=2 \mathrm{~cm})$ and the slightly overlapping cone-beam geometry $(\ell=7 \mathrm{~cm})$.

Also, as a comparison, we did simulations of the MCAT phantom with the dual-planar cone-beam geometry as illustrated in Fig. 1. The axial offset was $7 \mathrm{~cm}$ between the two point-sources which irradiated the two different heads. Using floods measured on the IRIX SPECT system and a numerical cone-beam projector, we again simulated two 1-minute acquisitions (each with $1.8 \mathrm{M}$ combined counts on the two heads), with one noise-free and another with Poisson noise.

\section{Transmission Measurements of an Anthropomorphic Phantom}

On the IRIX SPECT system with both of the Beacon transmission devices deployed, we performed a series of 4-minute flood and 1-minute transmission measurements of the Data Spectrum Anthropomorphic phantom (which is of $20.5 \mathrm{~cm}$ axial extent) using the two-camera heads opposed to the point sources. The axial locations of point-sources shifted by -10.5 , $-3.5,3.5,10.5 \mathrm{~cm}$ relative to the center of the field of view of the camera heads. Since we only have one Ba-133 point source per head, we summed the projection data from individual measurements on each of two camera heads to simulate the overlapping cone-beam projection data shown in Fig. 3.

\section{Reconstruction and Analysis of Overlapping Beam Data}

The 120-frame projections were equally divided into 30 subsets. We reconstructed the noise-free data using the OSTR algorithm for overlapping beams with weak regularization ( $\beta=0.1)$ and 50 iterations. To assess the accuracy of the reconstructions, region-of-interests (ROIs) were generated for soft tissue and lungs by thesholding the original MCAT phantom. The average values of the attenuation coefficients for soft tissue and lungs were calculated for each transaxial slice within the ROIs. Fig. 5 shows the portion of the soft tissue and lung ROIs within two example transaxial slices. The ROIs covered multiple slices bounded by the axial coverage for the given cone-beam geometry. For both the severely overlapping cone-beam geometry and the slightly overlapping cone-beam geometry, the axial coverage was determined respectively from the slice range in which the average values of soft tissue and lungs were accurately reconstructed (within $2 \%$ relative error).

We optimized the scaling factor $\beta$ and the number of iterations for reconstruction of the noisy simulation with the severely overlapping cone-beam geometry $(\ell=2 \mathrm{~cm})$ by calculating the root-mean-square (RMS) error of the attenuation coefficients 

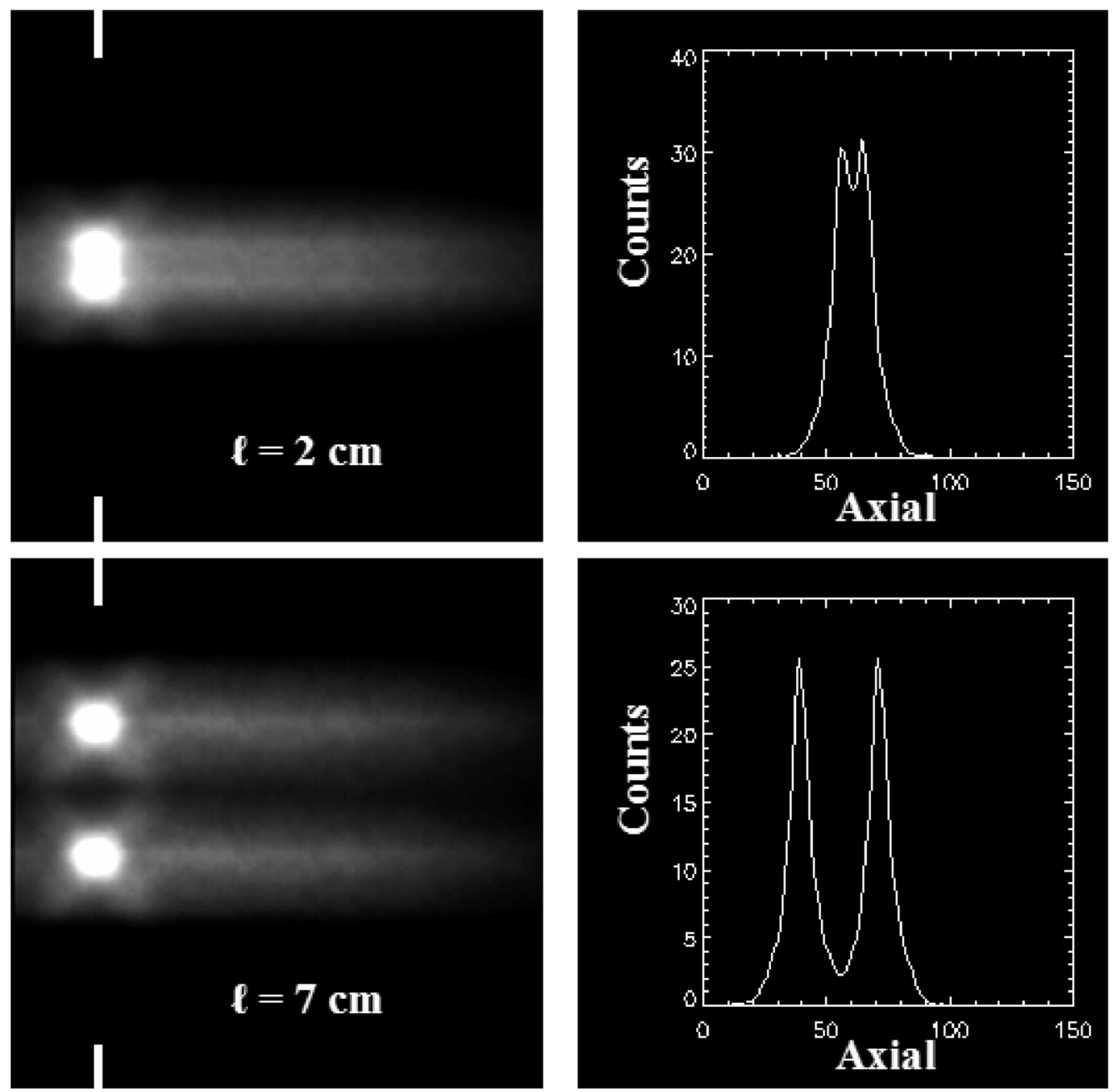

Fig. 4. The floods for the overlapping cone-beam geometry as shown in Fig. 3 were measured on the IRIX Beacon system for $1=2 \mathrm{~cm}$ (upper) and $1=7 \mathrm{~cm}$ (lower). In each case the axial profile through the bars shown on floods (left) is also plotted (right). Note that the floods from two point-sources severely overlap for the $1=2 \mathrm{~cm}$ case.

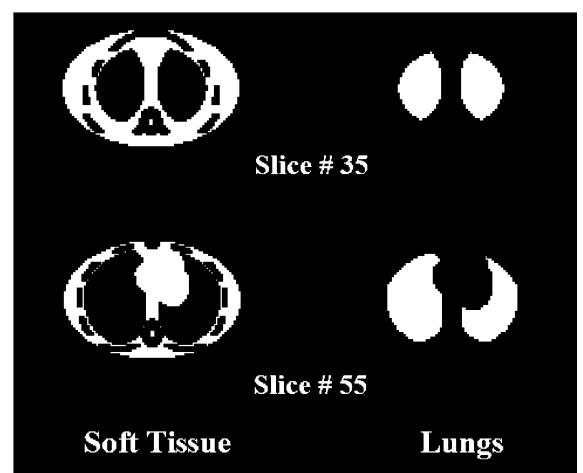

Fig. 5. Example transaxial slices from the region of interest (ROI) for soft tissue (left) and lungs (right) for the MCAT phantom. Note area not included in either ROI is bone or area outside of the phantom.

for soft tissue and lungs within the ROIs determined from the noise-free reconstruction. The RMS error in the ROI K is defined as [13]

$$
\mathrm{RMS}_{K}=\sqrt{\frac{\sum_{i}^{i \in \mathrm{ROI}_{K}}\left(\mu_{i}-\mu_{i, K}\right)^{2}}{N_{K}-1}},
$$

where $\mathrm{K}$ is either soft tissue or lungs, the summation is performed over all pixels in the ROI K, $\mu_{i}$ is the reconstructed attenuation coefficient at pixel $i, \mu_{i, K}$ is its true value, and $N_{K}$ is the number of pixels in the ROI K. Thereafter we applied these parameters ( $\beta$ and the iteration number) in reconstructing noisy data simulated or measured in the 1-minute acquisitions with the slightly overlapping cone-beam geometry $(\ell=7 \mathrm{~cm})$. Our reason for using the same $\beta$ for different overlapping conebeam geometries is that choice of the scaling factor $\beta$ should be mainly influenced by noise level and imaging time. To evaluate this hypothesis, we compared the RMS errors of the OSTR reconstructions using the same $\beta$ and iteration number for the severely overlapping cone-beam geometry $(\ell=2 \mathrm{~cm})$ and for the slightly overlapping cone-beam geometry $(\ell=7 \mathrm{~cm})$. We expected that these RMS errors should be of the same order of magnitude. We also expected that the RMS errors will be greater for the noisy simulation than for the noise-free simulation. This is because we know noise degrades the reconstruction accuracy, and inclusion of a stronger prior to control noise may cause a tradeoff between the smoothness and resolution of the reconstruction. To check whether these increases of the RMS errors were caused by the failure of the OSTR algorithm in noisy data, 


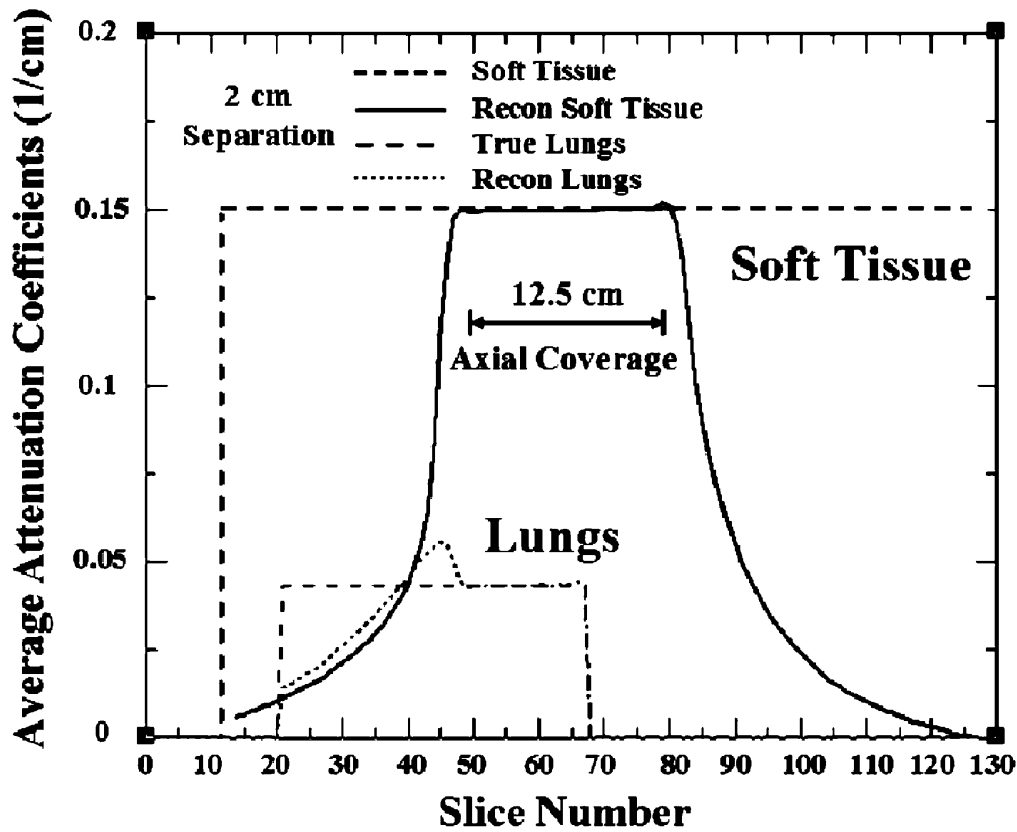

Fig. 6. The average values of the attenuation coefficients in the soft tissue and lung ROIs reconstructed from the noise-free MCAT phantom simulation with the severely overlapping geometry $(1=2 \mathrm{~cm}$ in Fig. 3$)$ are plotted with the true values versus the slice number. The axial coverage was determined as the slice range in which both soft tissue and lungs were reconstructed within $2 \%$ accuracy.

we did noise-free and noisy simulations of the MCAT phantom using the dual-planar cone-beam geometry plotted in Fig. 1.

We reconstructed the transmission measurements of the Anthropomorphic phantom (described in previous Section C) with the OSTR algorithm for overlapping beams using the optimal $\beta$ and iteration number determined from the MCAT phantom simulations as described above.

\section{RESULTS}

\section{A. The OSTR Algorithm for Overlapping Cone-Beams Using MCAT Phantom Simulations}

As shown in Fig. 6, a $12.5 \mathrm{~cm}$ axial coverage was determined as the slice range in which both soft tissue and lungs were reconstructed with an average value of the attenuation coefficient within $2 \%$ of that of the true value for the severely overlapping cone-beam geometry in the noise-free simulations. Note the significant deviation in the average values of the attenuation coefficient in the slices superior to the indicated range for axial coverage for both the soft tissue and lungs and inferior to this range for the soft tissue. The RMS error for the soft tissue was $\mathrm{RMS}_{\mathrm{st}}=8.0 \times 10^{-4} \mathrm{~cm}^{-1}$, or $0.5 \%$ of the true value, and for the lungs was $\mathrm{RMS}_{l}=7.6 \times 10^{-4} \mathrm{~cm}^{-1}$, or $1.8 \%$ of the true value within the determined range of axial coverage. These small RMS errors illustrate the success of the OSTR algorithm for reconstructing even the severely overlapping cone-beam geometry in the noise-free case with a $\beta=0.1$.

Plots of the RMS errors of the reconstructed attenuation coefficients for soft tissue and lungs within the axial coverage for the severely overlapping cone-beam geometry for the noisy MCAT phantom simulations are shown in Fig. 7. An iteration number of 25 was chosen since more iterations only slightly decreased the RMS errors. A $\beta=50$ was chosen based on the minimum
RMS error of the attenuation coefficient for soft tissue. Thus an iteration number of 25 and a $\beta=50$ were used when reconstructing the 1-minute noisy simulations and 1-minute measurements with the anthropomorphic phantom.

Compared to the noise-free simulation, the RMS errors in the noisy simulation increased to $\mathrm{RMS}_{\mathrm{st}}=0.013 \mathrm{~cm}^{-1}$ or $8.3 \%$ of the true value for soft tissue and $\mathrm{RMS}_{l}=0.022 \mathrm{~cm}^{-1}$ or $50 \%$ of the true value for lungs, each calculated within the $12.5 \mathrm{~cm}$ axial coverage defined in Fig. 6. Selected transaxial slices and profiles from them are plotted in Figs. 8 and 9.

After investigating the OSTR algorithm for the severely overlapping cone-beam geometry, we investigated its use in reconstructing the noise-free and noisy transmission projections simulated with the slightly overlapping cone-beam geometry $(\ell=$ $7 \mathrm{~cm}$ in Fig. 3). As shown in Fig. 10, a $26.3 \mathrm{~cm}$ axial coverage was measured on the plots of the average values versus the slice number as having a deviation of less than $2 \%$ from the true values of the attenuation coefficient in the soft tissue and lungs for the noise-free case. The RMS errors of the attenuation coefficients were calculated in the 3D volume within this axial coverage. For soft tissue the RMS error was $\mathrm{RMS}_{\mathrm{st}}=5.0 \times 10^{-4}$ $\mathrm{cm}^{-1}$ or $0.33 \%$ of the true value, and for lungs the RMS error was $\mathrm{RMS}_{l}=4.1 \times 10^{-4} \mathrm{~cm}^{-1}$ or $0.95 \%$ of the true value. The projections simulated with Poisson noise were reconstructed with 25 iterations of the OSTR algorithm for overlapping beams and with $\beta=50$ as determined in the optimization step for the severely overlapping case. The average values of the attenuation coefficients for soft tissue and lungs are plotted versus the axial slice number in Fig. 10. There it can be seen that, in the portion of the body containing the lungs, the average value of the attenuation coefficient in the soft tissue is under estimated, and that in the lung region is over estimated. These biases result from the blurring of the soft tissue and lung regions as illustrated 

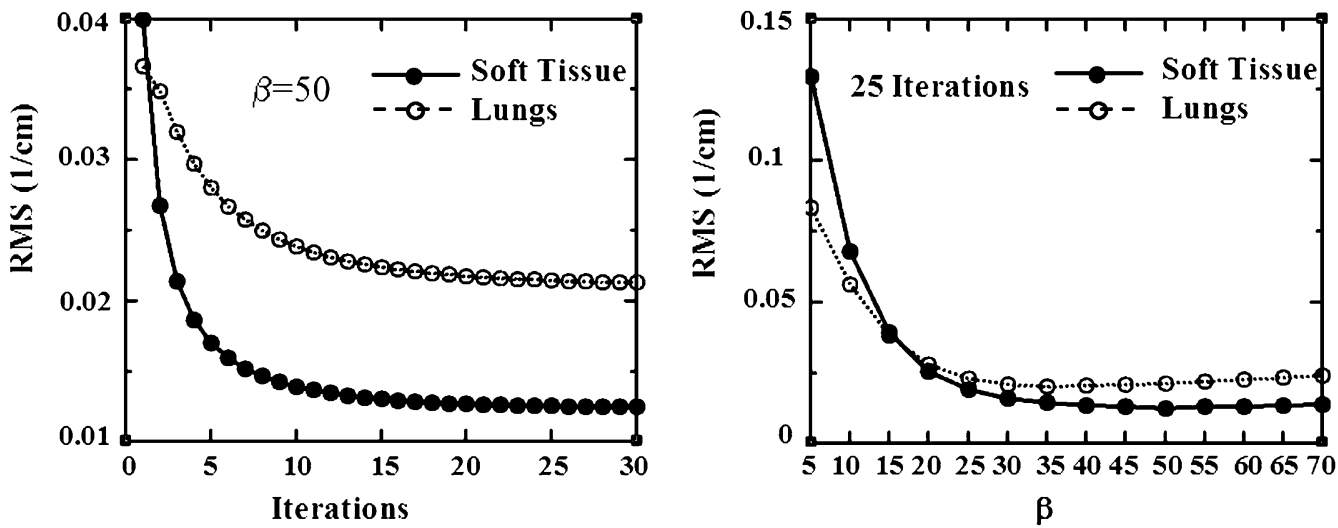

Fig. 7. The RMS errors of the attenuation coefficients for soft tissue and lungs within the region of axial coverage are plotted versus iteration number (left) and $\beta$ (right) for reconstructing the noisy transmission projections of the simulated 1-minute acquisition with the severely overlapping cone-beam geometry $(1=2 \mathrm{~cm})$.

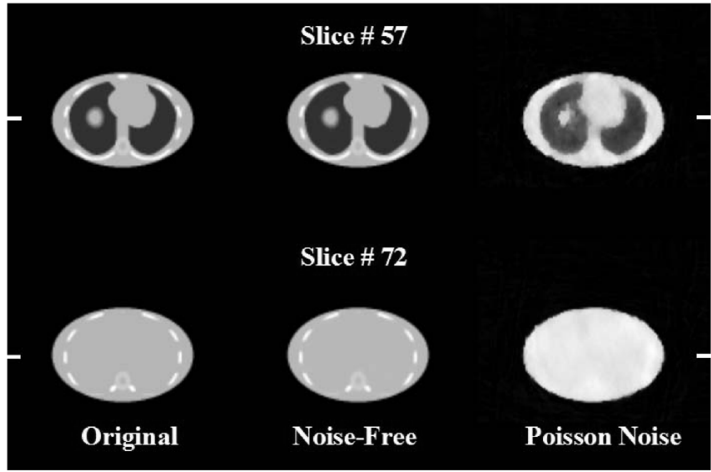

Fig. 8. Simulations with the severely overlapping cone-beam geometry $(1=2$ $\mathrm{cm})$. Two slices of the original MCAT phantom (left), the reconstructions with 50 iterations of the OSTR algorithm with $\beta=0.1$ from noise-free projections (middle), and the reconstructions with 25 iterations of the OSTR with $\beta=50$ from the noisy projections (right). The short bars on the two sides of the figure indicate the levels of the profiles plotted in Fig. 9.

in Fig. 9 for the severely overlapping geometry. Compared to the noise-free case, the RMS errors in the noisy simulation increased to $\mathrm{RMS}_{\mathrm{st}}=0.012 \mathrm{~cm}^{-1}$ or $8.2 \%$ of the true value for soft tissue, and $\mathrm{RMS}_{l}=0.025 \mathrm{~cm}^{-1}$ or $59 \%$ of the true value for lungs.

The dual-planar noise-free MCAT cone-beam data were reconstructed by 25 iterations of the OSTR algorithm with weak regularization $(\beta=0.1)$. From the noise-free reconstruction we determined the axial coverage was about $14.6 \mathrm{~cm}$ as illustrated in Fig. 11. Within the axial coverage the RMS errors of the attenuation coefficients were calculated as $\mathrm{RMS}_{\mathrm{st}}=6.6 \times 10^{-4}$ $\mathrm{cm}^{-1}$ or $0.44 \%$ of the true value for soft tissue and $\mathrm{RMS}_{l}=$ $5.4 \times 10^{-4} \mathrm{~cm}^{-1}$ or $1.3 \%$ of the true value for lungs for the noise free case. In [3] we have determined the optimal value $(\beta=80)$ of the scaling factor that provided the lowest RMS errors for soft tissue attenuation coefficients. This resulted in RMS errors of the attenuation coefficients for soft tissue of $\mathrm{RMS}_{\mathrm{st}}=0.014$ $\mathrm{cm}^{-1}$ or $9.6 \%$ of the true value and for lungs of $\mathrm{RMS}_{l}=0.027$ $\mathrm{cm}^{-1}$ or $64 \%$ of the true value. These RMS errors were comparable with the RMS errors we obtained with the slightly overlapping cone-beam geometry. This indicates that the increases in the RMS errors in the noisy simulations were likely not caused
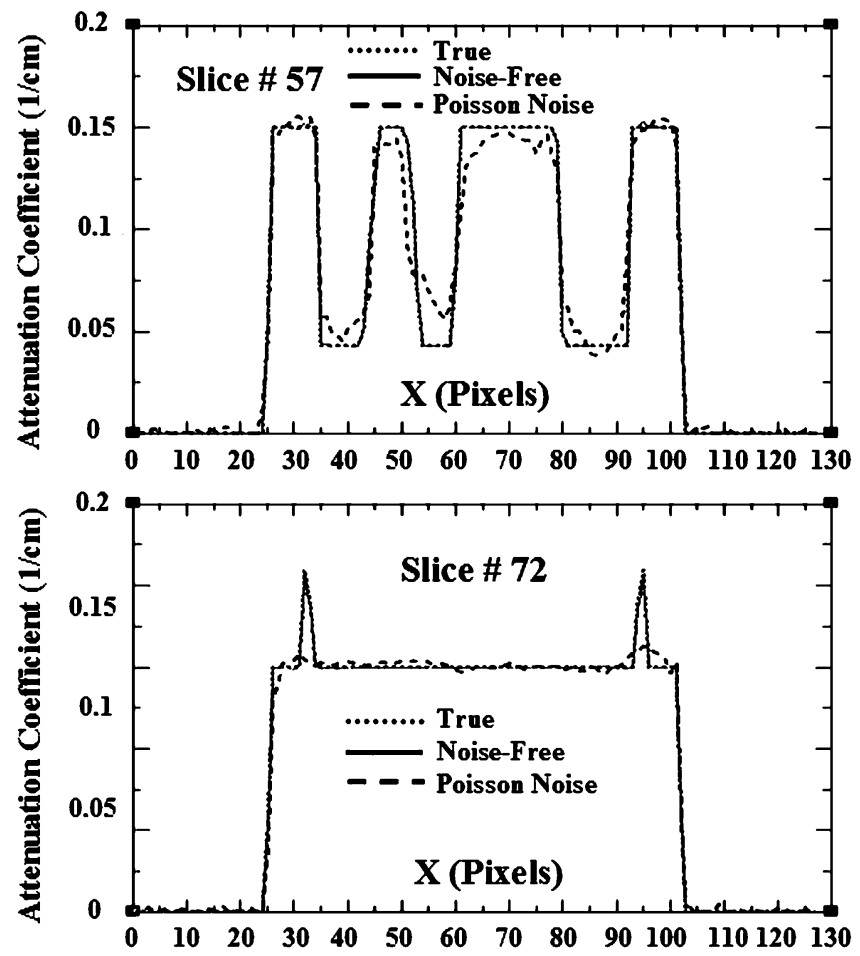

Fig. 9. The horizontal profiles of the attenuation coefficient are plotted through the center of the slice \#57 (upper) and the slice \#72 (lower). Note that the profiles of the noise-free reconstruction (solid line) almost coincide with the true profiles (dotted line) making it difficult to tell the two apart. The dashed line is for the profiles reconstructed from the noisy projections.

by the failure of the reconstruction algorithm in the overlapping beam cases.

We summed the projection data from individual measurements on the IRIX Beacon system to simulate the projection data for the slightly overlapping cone-beam geometry shown in Fig. 3. Reconstruction using 25 iterations of the OSTR algorithm for overlapping beams is shown in Fig. 12. The regularization factor used was $\beta=50$. For comparison, the reconstruction for the dual-planar cone-beam geometry where there is just one source irradiating each camera-head (Fig. 1) is shown in Fig. 13. The improved axial coverage with the overlapping cone-beam geometry is evident. About $15 \mathrm{~cm}$ axial coverage 


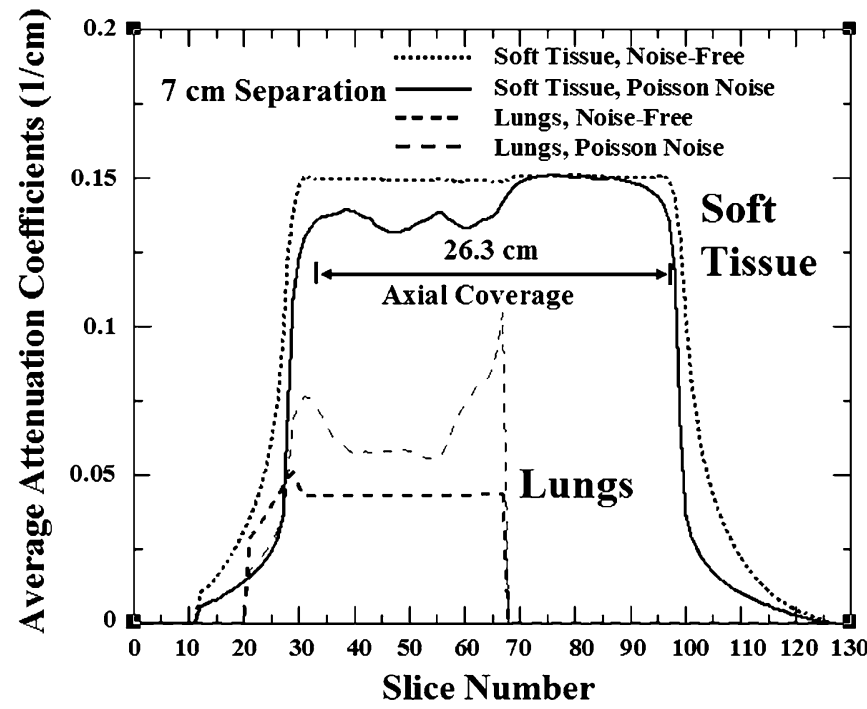

Fig. 10. The average values of the attenuation coefficients in the soft tissue and lung ROIs reconstructed from the noise-free and noisy projections simulated with the slightly overlapping cone-beam geometry $(1=7 \mathrm{~cm}$ in Fig. 3$)$, the average values of attenuation coefficients for soft tissue and lungs are plotted versus the slice number. The axial coverage was determined from the noise-free simulation.

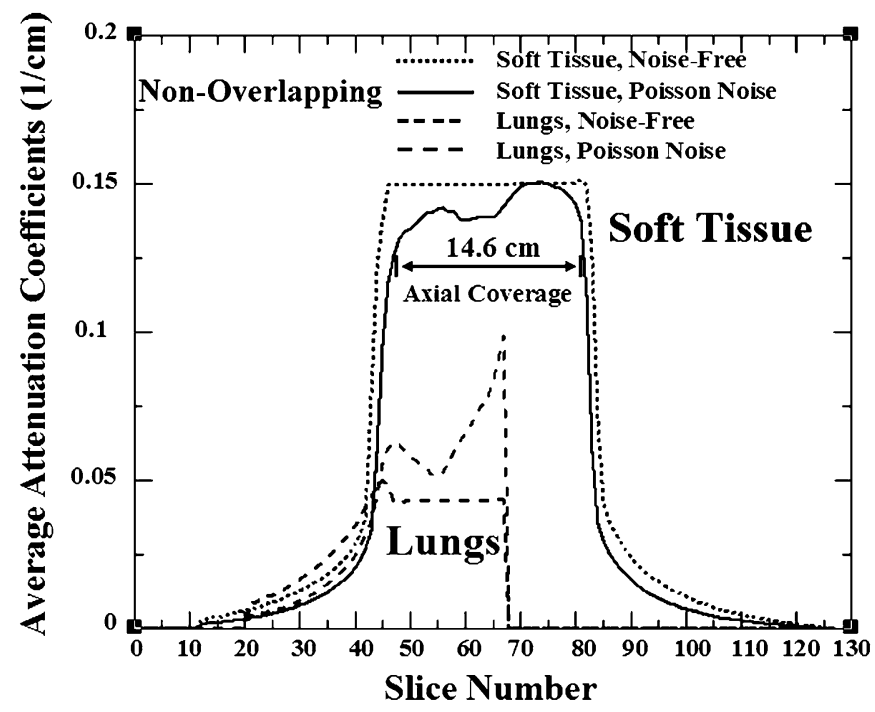

Fig. 11. From the reconstructions of the noise-free and noisy projections simulated with the dual-planar cone-beam geometry shown in Fig. 1, the average values of attenuation coefficients for soft tissue and lungs are plotted versus the slice number. The axial coverage was determined from the noise-free simulation.

for the dual-planar cone-beam geometry was measured on the reconstructed map for anthropomorphic phantom. This was in good agreement with the MCAT phantom simulation. The entire axial extent of the anthropomorphic phantom was reconstructed for the slightly overlapping cone-beam geometry, indicating the axial coverage of the system was greater than the $20.5 \mathrm{~cm}$ extent of the phantom.

We summarized our simulation and measurement results in Table I.

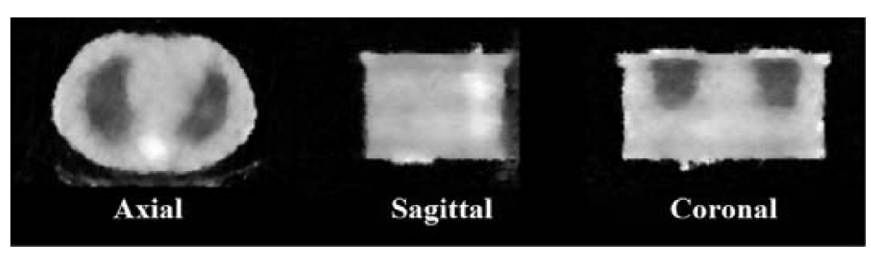

Fig. 12. The anthropomorphic phantom reconstructed from 1-minute acquisition using the overlapping geometry $(1=7 \mathrm{~cm})$ shown in Fig. 3.

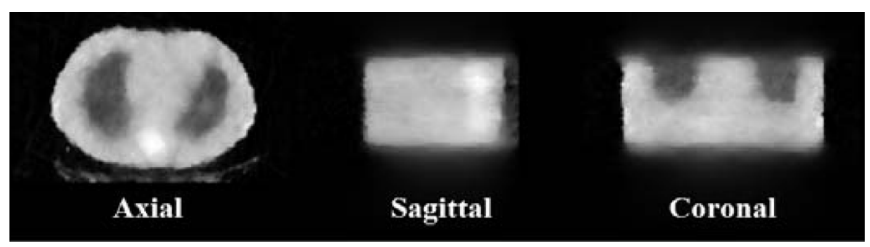

Fig. 13. The anthropomorphic phantom reconstructed from 1-minute acquisition using the dual-planar geometry shown in Fig. 1. Note that difference in axial extent between Figs. 12 and 13 appears less that one might expect due the limited axial extent of the anthropomorphic phantom which is $20.5 \mathrm{~cm}$.

TABLE I

The Summary of Simulations and MEASUREMENTS IN THIS STUdy For SEVERELY OVERLAPPING $(1=2 \mathrm{~cm})$, SLIGHTLY OVERLAPPING $(1=7 \mathrm{~cm})$, AND NO OVERLAP (NO) TRANSMISSION GEOMETRIES

\begin{tabular}{|c|c|c|c|c|c|}
\hline & $\begin{array}{c}\text { Trans } \\
\text { Geometry }\end{array}$ & $\begin{array}{c}\text { Counts } \\
\text { on Two } \\
\text { Cameras } \\
\text { (M) }\end{array}$ & $\beta$ & $\begin{array}{c}\text { Axial } \\
\text { Coverage } \\
(\mathrm{cm})\end{array}$ & $\begin{array}{l}\text { RMS } \\
\text { Errors } \\
\text { Soft } \\
\text { Tissue, } \\
\text { Lungs }\end{array}$ \\
\hline \multirow{6}{*}{$\begin{array}{c}\text { Simulations } \\
\text { (Noisy -N, } \\
\text { Noise Free - } \\
\text { NF) }\end{array}$} & $\mathrm{l}=2 \mathrm{~cm}, \mathrm{NF}$ & 3.6 & 0.1 & 12.5 & $\begin{array}{l}0.5 \% \\
1.8 \% \\
\end{array}$ \\
\hline & $\mathrm{l}=2 \mathrm{~cm}, \mathrm{~N}$ & 3.6 & 50 & 12.5 & $8.3 \%, 50 \%$ \\
\hline & $\mathrm{l}=7 \mathrm{~cm}, \mathrm{NF}$ & 3.6 & 0.1 & 26.3 & $\begin{array}{l}0.33 \% \\
0.95 \% \\
\end{array}$ \\
\hline & $\mathrm{l}=7 \mathrm{~cm}, \mathrm{~N}$ & 3.6 & 50 & 26.3 & $\begin{array}{l}8.2 \%, \\
59 \% \\
\end{array}$ \\
\hline & $\mathrm{NO}, \mathrm{NF}$ & 1.8 & 0.1 & 14.6 & $\begin{array}{c}0.44 \% \\
1.3 \% \\
\end{array}$ \\
\hline & $\mathrm{NO}, \mathrm{N}$ & 1.8 & 80 & 14.6 & $\begin{array}{c}9.6 \%, \\
64 \% \\
\end{array}$ \\
\hline \multirow[t]{2}{*}{ Measurements } & $1=7 \mathrm{~cm}$ & 3.8 & 50 & $>20.5 *$ & Unknown \\
\hline & $\mathrm{NO}$ & 1.7 & 80 & $\sim 15$ & Unknown \\
\hline
\end{tabular}

\section{DISCUSSION}

In the transaxial plane, the asymmetric cone-beam geometry can be approximated by the asymmetric fan beam geometry for the slice directly opposite a point source. As one moves away to either side of this slice the data sufficiency condition is not satisfied. We have show in a previous publication [2] through eigen analysis, reconstruction of simulations of transmission imaging of a digital Defrise phantom, and experimental acquisitions of an anthropomorphic phantom that an artifact free and fairly accurate reconstruction of attenuation maps can be obtained for transaxial slices extending over a limited region axially to either side of the location of the point source. With a dual-planar geometry employing two point sources separated axially and irradiating different gamma-camera heads we were able to obtain an artifact-free region of approximately $15 \mathrm{~cm}$ in axial extent. Herein we investigated extending the axial coverage by 
having multiple-point sources separated axially irradiating the same head. Within the axial coverage, the reconstruction was shown accurate with a less than $2 \%$ relative error in noise-free simulation.

We investigated the use of the OSTR algorithm for reconstruction of attenuation maps from the overlapping cone-beam irradiation fields for MCAT phantom simulations and experimental acquisitions of the Data Spectrum Anthropomorphic Phantom. We found that the algorithm was able to sort the overlapped counts correctly and reconstruct the attenuation map accurately for a $12.5 \mathrm{~cm}$ axial extent (Figs. 6, 8,9) in the case of severely overlapping geometry. For the noise-free case, the RMS errors of the attenuation coefficients for soft tissue $(0.5 \%$ of the true value) and lungs (1.8\% of the true value) were small. We optimized the iteration number and regularization parameter $\beta$ for noisy MCAT phantom simulation with the severely overlapping cone-beam geometry and applied these parameters to the less severely overlapping cone-beam geometry. The regularization parameter depends mainly on the noise level. That was the reason we applied the same $\beta$ to acquisitions or simulations with the same imaging time but with different imaging geometries. We found that the RMS errors $\left(\mathrm{RMS}_{\mathrm{st}}=0.012 \mathrm{~cm}^{-1}\right.$ for soft tissue, $\mathrm{RMS}_{l}=0.025 \mathrm{~cm}^{-1}$ for lungs) in the case of the noisy simulation with the slightly overlapping cone-beam geometry were comparable to the RMS errors $\left(\mathrm{RMS}_{\mathrm{st}}=0.013 \mathrm{~cm}^{-1}\right.$ for soft tissue, $\mathrm{RMS}_{l}=0.022 \mathrm{~cm}^{-1}$ for lungs) in the case of the severely overlapping cone-beam.

The threshold $\delta$ was set to $0.0025 \mathrm{~cm}^{-1}$ in this study, which was about $1 / 20$ of the attenuation coefficient of lungs at 140 $\mathrm{keV}$. We determined that with a smaller $\delta$ we started to see isolated hot spots with reconstruction. For the extremely low counts ( $\sim 3.6 \mathrm{M}$ on two heads, 1 minute acquisition time) simulated in this study, a strong regularization was needed to control the noise and reconstruct smooth attenuation maps. This resulted in the compromise between the smoothness and the resolution of the reconstruction. The degradation of resolution caused apparent increase of the RMS errors, since we used the large ROIs for soft tissue and lungs (Fig. 5). As a comparison we did simulations with the dual-planar cone-beam geometry which has no overlap. We determined that the RMS errors were on the same order as for the overlapping cone-beam geometry, which indicated that the loss of resolution was inherent in the low-count imaging, and not because of the failure of applying the OSTR algorithm to overlapping cone-beams. In our previous work [2] we determined that the attenuation maps obtained with the dualplanar cone-beam geometry could provide satisfactory attenuation compensation for emission imaging. Thus, we can infer that attenuation maps obtained with the overlapping cone-beam geometry would do the same since they had the similar image quality and wider axial coverage.

For the 4 point-source cone-beam system (two per head) with the optimal arrangement $(1=7 \mathrm{~cm}$ in Fig. 3$)$, the axial coverage was estimated as $26.3 \mathrm{~cm}$ from simulations. To obtain a wider axial coverage, more point-sources could be employed. In previous work [14], we investigated the feasibility of adapting the asymmetric cone-beam transmission imaging geometry to imaging systems which cannot rotate 360 degrees. For those systems with limited gantry rotation, a denser array of point- sources may help in reducing the artifacts caused by the limited angle reconstruction, because such a denser array approximates the fan-beam geometry more closely.

Overshoots are seen in the lung region in the noise-free simulations in Figs. 6, 10, and 11. These are at the edge of what we determined to be the useful axial-extent of reconstruction. We believe the overshoots are due to data sufficiency artifacts associated with use of cone-beam imaging, and a decrease in beam intensity associated with point-source collimation and the ability of the photons to penetrate the collimators on the camera heads. The soft tissue ROI region noise-free results show a falloff in the average attenuation coefficients at the same axial locations. Both lung and soft tissue regions then show a decrease in the average attenuation coefficients as the artifacts increase as one proceeds further axially. Notice in the figures that where the axial coverage extends below the end of the lungs in the abdominal region there is no overshoot for the noise-free simulations. When noise is included in the simulation, the lung ROI shows an increase in the average attenuation coefficient and the soft tissue ROI shows a decrease in the average attenuation coefficient for the slices where both are present. We believe this is due in part to a value of 50 as opposed to 0.1 being used for $\beta$ in the roughness penalty function, and the voxels of the two ROIs abutting each other as shown in Fig. 5. Thus the increased smoothing in the reconstruction when noise is included in the simulation causes a change in the average value of the ROIs when both lung and soft tissue are present in the slices.

\section{CONCLUSION}

Using numerical simulations of the MCAT phantom, we investigated application of the OSTR algorithm to the overlapping cone-beam transmission geometry. The algorithm was able to reconstruct accurately noise-free data simulated with the severely overlapping cone-beam geometry. Using noisy simulations we investigated the degradation of reconstruction accuracy by noise, and found the accuracy comparable to that of the dual-planar cone-beam geometry which has no overlap. Through simulations we also investigated the axial coverage, optimized reconstruction parameters, and successful applied these parameters to reconstruction of experimental transmission-measurements of the Data Spectrum anthropomorphic phantom. We determined that the overlapping cone-beam geometry can provide a substantial increase in axial coverage over that of the dual-planar cone-beam system. We concluded that axially overlapping cone-beam geometry can be used to design a fast transmission imaging system which covers the whole thorax.

\section{REFERENCES}

[1] G. L. Zeng, G. T. Gullberg, and P. E. Christian, "Asymmetric conebeam transmission tomography," IEEE Trans. Nucl. Sci., vol. 48, no. 1, pp. 117-124, Feb. 2002.

[2] B. Feng, M. A. King, G. L. Zeng, P. H. Pretorius, P. P. Bruyant, R. D. Beach, G. Boening, G. Jarkewicz, S. Cochoff, and D. Gagnon, "The estimation of attenuation maps for cardiac-spect using cone-beam imaging of high-energy photons through parallel-hole collimators," IEEE Trans. Nucl. Sci., vol. 51, pp. 2699-2704, Oct. 2004.

[3] B. Feng, J. A. Fessler, G. Boening, R. D. Beach, and M. A. King, "Penalized Likelihood reconstruction for dual-planar cone-beam transmission imaging," J. Nucl. Med., vol. 45, p. 104P, May 2004, (abstract). 
[4] B. Feng, H. P. Pretorius, P. P. Bruyant, G. Boening, R. D. Beach, J. A. Fessler, and M. A. King, "Impact on cardiac reconstruction of using attenuation maps reconstructed with less than complete rotation acquired by asymmetric cone-beam transmission imaging," J. Nucl. Med., vol. 45, p. 158P, May 2004, (abstract).

[5] D. Gagnon, C. H. Tung, G. L. Zeng, and W. Hawkins, "Design and early testing of a new medium-energy transmission device for attenuation correction in SPECT and PET," in Conf. Rec. 1999 IEEE Nuclear Science Symp., Seattle, WA, Oct. , pp. 24-30.

[6] D. S. Lalush and A. J. DiMeo, "A monte carlo investigation of dualplanar circular-orbit cone-beam SPECT," Phys. Med. Biol., vol. 47, pp. 4357-4370, 2002.

[7] D. S. Lalush and A. J. DiMeo, "An observer study evaluating dualplanar circular-orbit cone-beam brain SPECT," J. Nucl. Med., vol. 43, pp. 1578-1583, 2002.

[8] D. S. Lalush, "Fourier rebinning applied to multiplanar circular-orbit cone-beam SPECT," IEEE Trans. Med. Imag., vol. 18, no. 11, pp. 1076-1084, Nov. 1999.
[9] H. Erdoğan and J. A. Fessler, "Ordered subsets algorithms for transmission tomography," Phys. Med. Biol., vol. 44, pp. 2835-51, 1999.

[10] H. Erdoğan and J. A. Fessler, "Monotonic algorithms for transmission tomography," IEEE Trans. Med. Imag., vol. 18, no. 9, pp. 801-814, Sep. 1999.

[11] D. F. Yu, J. A. Fessler, and E. P. Ficaro, "Maximum likelihood transmission image reconstruction for overlapping transmission beams," IEEE Trans. Med. Imag., vol. 19, no. 11, pp. 1094-1105, Nov. 2000.

[12] P. J. Huber, Robust Statistics. New York: Wiley, 1981.

[13] A. Celler, K. L. Dixon, Z. Chang, S. Blinder, J. Powe, and R. Harrop, "Problems created in attenuation-corrected SPECT images by artifacts in attenuation maps: A simulation Study," J. Nucl. Med., vol. 46, pp. 335-343, 2005.

[14] B. Feng, P. H. Pretorius, P. P. Bruyant, G. Boening, R. D. Beach, H. C. Gifford, J. A. Fessler, and M. A. King, "Body-and-lungs-outline penalized-likelihood reconstruction of asymmetric cone-beam transmission data acquired with less than complete gantry rotation," J. Nucl. Cardiol., vol. 11, no. S3, Aug. 2004, (abstract). 